\title{
Association of RENAL nephrometry score with outcomes of minimally invasive partial nephrectomy
}

Jonathan S Ellison, Jeffrey S Montgomery, Khaled S Hafez, David C Miller, Chang He, J Stuart Wolf Jr and Alon Z Weizer

Department of Urology, University of Michigan, Ann Arbor, Michigan, USA

Abbreviations \& Acronyms
ARF = acute renal failure
ASA = American Society
of Anesthesiologists
BMI = body mass index
CLPN = conventional
laparoscopic partial
nephrectomy
EBL = estimated blood loss
eGFR = estimated
glomerular filtration rate
HAPN = hand-assisted
laparoscopic partial
nephrectomy
LOS = length of stay
MI-NSS = minimally
invasive nephron-sparing
surgery
PN = partial nephrectomy
RAPN = robotic-assisted
partial nephrectomy
RENAL = Radius,
Exophytic, Nearness,
Anterior, Location
WIT = warm ischemia time

Correspondence: Alon Z

Weizer M.D., M.S., Department of Urology, University of Michigan, 7312 CCC, 1500 E Medical Center Dr, SPC 5946, Ann Arbor, MI 48109, USA. Email: aweizer@med.umich.edu

Received 1 July 2012; accepted 2 October 2012.

Online publication 6 November 2012
Objective: To evaluate the predictive value of the Radius, Exophytic, Nearness, Anterior, Location nephrometry scoring system and to investigate the influence of its individual components on perioperative outcomes of minimally invasive partial nephrectomy.

Methods: Consecutive laparoscopic partial nephrectomy $(n=189)$ and robotic partial nephrectomy $(n=109)$ cases from 2007 through 2011 were retrospectively reviewed from our prospectively maintained database. Urological surgeons assigned nephrometry scores, excluding cases without images available for review. The association of nephrometry score categories and individual components of the score to perioperative outcomes were assessed.

Results: No differences were observed in preoperative characteristics of low $(n=135)$, intermediate $(n=155)$ and high $(n=8)$ nephrometry groups. Higher nephrometry score was associated with an increased length of stay, estimated blood loss and warm ischemia time. Higher nephrometry scores were also associated with a greater proportion of major complications $(P<0.001)$. Distance to the renal sinus had the greatest impact on perioperative outcomes including operative and ischemic times, estimated blood loss, complications and length of stay.

Conclusions: The Radius, Exophytic, Nearness, Anterior, Location nephrometry score has value as a predictive tool for perioperative outcomes of minimally invasive partial nephrectomy. Distance to the renal sinus seems to have the greatest association with outcomes. Using these findings, clinicians will be better able to counsel patients regarding anticipated perioperative outcomes of minimally invasive partial nephrectomy.

Key words: kidney neoplasms, laparoscopy, nephron-sparing surgery, renal cell carcinoma, robotics.

\section{Introduction}

Although recent trends have shown an increase in the use of minimally invasive and nephron-sparing approaches to the management of small renal masses, these techniques can be challenging and might pose a greater risk of perioperative morbidity to the patient. ${ }^{1}$ Existing data on the perioperative outcomes of minimally invasive nephron sparing surgery (MI-NSS) is derived from single or multi-institutional case series. Only a handful of studies have examined the impact of individual tumor and patient factors on outcomes of MI-NSS. ${ }^{2}$ Understanding the potential morbidity of a particular approach to the management of a small renal mass based on individual patient factors could aid in making treatment decisions.

Recently, several renal tumor scoring systems have been developed to provide a common nomenclature to better describe renal masses and objectively predict the difficulty of resection. The RENAL nephrometry score has the advantage of being adaptable and reproducible, determined by five anatomical characteristics of the mass. ${ }^{3}$ Several series have evaluated the impact of nephrometry on medical decision-making, perioperative outcomes and short-term oncological outcomes; ${ }^{4-7}$ however, a recent series failed to show an association 
between nephrometry and perioperative outcomes. ${ }^{8}$ Meanwhile, no study has investigated the impact of the individual components of the nephrometry score on outcomes.

We sought to externally validate the association of RENAL nephrometry with perioperative outcomes using a large single institution cohort of patients undergoing MI-NSS. We also evaluated the significance of individual components of the scoring system on perioperative outcomes in order to offer insight into the value of these individual components.

\section{Methods}

\section{Patient selection}

All patients undergoing either CLPN, HAPN or RAPN for suspected malignancy from January 2007 to January 2011 were identified in our single-institution, prospectively maintained, Institutional review board-approved database and retrospectively reviewed. Conversions to radical nephrectomy were included (2 CLPN cases converted because of hemorrhage, and three RAPN cases converted for oncological control).

\section{RENAL nephrometry}

RENAL nephrometry scores were assigned as described by Kutuzov and Uzzo. ${ }^{3}$ The numerical total is a sum of individual tumor characteristics, each assigned a point from $1-3$, where a lower score indicates a less complex tumor. Included in this sum are radius $(\leq 4 \mathrm{~cm}, 4-7 \mathrm{~cm}$, and $\geq 7 \mathrm{~cm}$ ), endophytic nature of the tumor ( $\geq 50 \%$ exophytic, $<50 \%$ exophytic or endophytic), nearness to the renal sinus ( $\leq 4 \mathrm{~mm}, 4-7 \mathrm{~mm}$, or $\geq 7 \mathrm{~mm}$ ) and polar location of the tumor ( $\leq 50 \%$ crossing the interpolar lines, $>50 \%$ crossing the interpolar lines, $100 \%$ within the polar lines). The alphabetical indicators include $\mathrm{A}, \mathrm{P}$ or $\mathrm{x}$ for anterior, posterior or indeterminate tumors, respectively. Hilar tumors are defined as tumors that abut the renal hilum.

\section{Data acquisition}

Chart and imaging reviews were retrospectively carried out by four study investigators for procedures carried out before May 2010, and were captured prospectively from that point forward. Recent studies have suggested good interobserver reliability of nephrometry scoring. ${ }^{9,10}$ If imaging was unavailable, the patient was excluded from analysis. Tumors were stratified by RENAL score into low (4-6 points), intermediate (7-9 points) or high (10-12 points) groups. ${ }^{3}$ Complications were categorized using the Clavien Classification system. ${ }^{11}$ Clavien scores less than or equal to 2 were considered "minor", whereas those greater than 2 were considered "major" complications. Both early (within 30 days of surgery) and late (after 30 days of surgery) complications were included. Intraoperative complications were events requiring altered intraoperative management (i.e. damage to viscous or vascular structures requiring repair or hemorrhage requiring intraoperative blood transfusion). Additional demographic/patient characteristics (age at operation, BMI, ASA score), perioperative information (WIT, LOS, EBL and operative time) and pathological data were abstracted from the database. EGFR was calculated using the Modification of Diet in Renal Disease equation, ${ }^{12}$ utilizing the last creatinine on record during follow up, with a mean follow up of 9 months.

\section{Surgical technique}

All procedures were carried out by one of eight attending urological surgeons at a single academic institution. Robotic procedures were carried out using the DaVinci surgical system (Intuitive Surgical, Sunnyvale, CA, USA). Surgical approach (CLPN, HAPN, RAPN) was determined at the discretion of the attending surgeon. Our institutional algorithm for management of renal masses at the time of minimally invasive partial nephrectomy with regards to hilar clamping, management of the tumor bed and transperitoneal or retroperitoneal approach has been previously described. ${ }^{13}$ Standardized care pathways were used to manage patients postoperatively.

\section{Data analysis}

Preoperative characteristics, perioperative outcomes and complications were compared among "low" and "intermediate" complexity tumors. "High" complexity tumors were excluded from categorical analysis of outcomes given low numbers, but an additional analysis utilizing all strata of nephrometry as a continuous variable in order to minimize the limitations of a small number of "high" complexity tumors was carried out, with results computed by linear regression. A sub-analysis of groups separated by surgical approach (RAPN, CLPN and HAPN) was also carried out. Bivariate comparisons of preoperative and perioperative characteristics and complications were carried out using $t$-test, Kruskal-Wallis test or Mantel-Haenszel $\chi^{2}$-test. Multivariable comparisons evaluating individual components of the RENAL score were explored by including all individual components in the starting model. Final models were then constructed using a backwards selection method. All statistical tests were two-tailed and carried out at the 5\% significance level. Analyses were carried out using the SAS V9.13 system (SAS Institute, Cary, NC, USA).

\section{Results}

A total of 298 out of 320 possible patients had all necessary data for analysis, including 109 RAPN, 36 HAPN and 153 
Table 1 Preoperative characteristics by RENAL nephrometry score

\begin{tabular}{lrrr}
\hline & Low $(n=135)$ & Intermediate $(n=155)$ & $P$ \\
\hline Age at operation (years) & $56 \pm 13.9$ & $58 \pm 12.6$ & 0.18 \\
BMI & $29.7 \pm 7.54$ & $30.2 \pm 6.02$ & 0.59 \\
ASA (median) & $84(62 \%)$ & $94(61 \%)$ & 0.81 \\
$1-2$ & $51(38 \%)$ & $61(39 \%)$ & 0.71 \\
$3-4$ & $84.2 \pm 23.5$ & $83.4 \pm 21.9$ & 0.10 \\
eGFR & $21(15.5 \%)$ & $20(12.9 \%)$ & \\
eGFR $<60$ & & &
\end{tabular}

Table 2 Intraoperative and postoperative outcomes by RENAL nephrometry score

\begin{tabular}{|c|c|c|c|}
\hline Intraoperative outcomes & $\begin{array}{l}\text { Low } \\
(n=135)\end{array}$ & $\begin{array}{l}\text { Intermediate } \\
(n=155)\end{array}$ & $P$ \\
\hline Estimated Blood Loss (mL) (median and range) & $150(5-1700)$ & $300(10-9000)$ & 0.0019 \\
\hline Warm Ischemia Time (min) & $14.5 \pm 13.4$ & $28.2 \pm 13.0$ & 0.0001 \\
\hline Operative Time (min) & $162 \pm 57.8$ & $207 \pm 61.3$ & 0.001 \\
\hline Transfusion requirement & $5(3.7 \%)$ & $15(9.7 \%)$ & 0.0617 \\
\hline Positive margins & $11(12 \%)$ & $6(4 \%)$ & 0.0356 \\
\hline RAPN & $41(30 \%)$ & $63(41 \%)$ & 0.0856 \\
\hline CLPN + HAPN & $94(70 \%)$ & $92(59 \%)$ & \\
\hline Postoperative outcomes & Low & Intermediate & $P$ \\
\hline Length of Stay (days) & $2.2 \pm 1.63$ & $3.1 \pm 2.5$ & 0.0007 \\
\hline eGFR & $84.7 \pm 23.8$ & $80.0 \pm 25.3$ & 0.1042 \\
\hline eGFR $<60$ & 17 (12.6\%) & 27 (17.4\%) & 0.325 \\
\hline New onset eGFR $<60$ & $4(3.0 \%)$ & $10(6.5 \%)$ & 0.1725 \\
\hline
\end{tabular}

Values presented as mean \pm SD unless otherwise noted.

CLPN. The average nephrometry score for the entire cohort was 6.6. There were 135 "low", 155 "intermediate" and eight "high" nephrometry score tumors. A total of 28 tumors qualified as hilar. Preoperative characteristics, including age at operation, BMI, ASA score, preoperative eGFR and number of patients with stage 3 or greater chronic kidney disease (eGFR <60) were similar among groups, as seen in Table 1. RAPN utilization increased with increasing nephrometry score, accounting for 30\%, 41\% and 63\% of "low", "intermediate" and "high" tumors, respectively $(P=0.05)$.

Intraoperative and postoperative outcomes are detailed in Table 2. EBL, WIT and operative time were significantly greater in the "intermediate" group as compared with the "low" group. Likewise, intraoperative complications were significantly greater in the "intermediate" group $(n=17)$ as compared with the "low" group $(n=2 ; P=0.0038)$. Evaluation of the RENAL score as a continuous variable confirmed no difference in preoperative parameters. Linear regression of postoperative outcomes showed significant differences in LOS $(R=0.1994 ; \quad P=0.0008)$, EBL $(R=0.1486 ; \quad P=0.0108)$, operative time $(R=0.3618$, $P<0.0001)$ and WIT $(R=0.5065, P<0.0001)$.

Postoperatively, LOS increased significantly as the nephrometry score increased $(P=0.003)$. There was no difference in the postoperative eGFR, number of patients with eGFR less than $60 \mathrm{mg} / \mathrm{mL} / 1.73 \mathrm{~m}^{2}$ or the number of patients who developed eGFR less than $60 \mathrm{mg} / \mathrm{mL} / 1.73 \mathrm{~m}^{2}$ postoperatively. The distance to the renal sinus was independently associated with longer $\operatorname{LOS}(P<0.0001)$.

Table 3 summarizes complications among the nephrometry groups. Major complications comprised $25 \%$ of all complications in the "intermediate" group and just $7 \%$ of all complications in the "low" group, with the remainder in each group being minor complications $(P=0.013)$. "Other" complications accounted for a large proportion of all complications, and included rhabdomyolysis, ureteral injury, urinary retention, ileus, urinary tract infection, Clostridium difficile infection, partial small bowel obstruction, unrecog- 


\begin{tabular}{|c|c|c|c|}
\hline Categorical variables & Low & Intermediate & $P$ \\
\hline \multicolumn{4}{|l|}{$\begin{array}{l}\text { Severity of } \\
\text { complication }\end{array}$} \\
\hline Minor & 49 (93\%) & $75(75 \%)$ & $<0.0001$ \\
\hline Major & $4(7 \%)$ & $25(25 \%)$ & \\
\hline \multicolumn{4}{|l|}{ Type of complication } \\
\hline Wound & 09 (17\%) & $7(7 \%)$ & 0.1288 \\
\hline Nerve & $4(7 \%)$ & $0(0 \%)$ & 0.008 \\
\hline Pulmonary & $4(7 \%)$ & $17(17 \%)$ & 0.3145 \\
\hline Cardiac & $2(4 \%)$ & $11(11 \%)$ & 0.2663 \\
\hline ARF & $1(2 \%)$ & $5(5 \%)$ & 0.5974 \\
\hline Urine leak & $2(4 \%)$ & $4(4 \%)$ & 0.9384 \\
\hline Other & $28(53 \%)$ & $39(39 \%)$ & 0.196 \\
\hline Hemorrhage & $3(6 \%)$ & $17(17 \%)$ & 0.1088 \\
\hline $\begin{array}{l}\text { Overall complication } \\
\text { rate (no. } \\
\text { patients with a } \\
\text { complication) }\end{array}$ & $43(32 \%)$ & $71(45 \%)$ & 0.044 \\
\hline
\end{tabular}

nized bowel injury, common bile duct injury, anemia, renal infarction, fever, diarrhea, emesis, hypertension, uncontrolled postoperative pain, acalculous cholecystitis, constipation, altered mental status, epididymitis, gout flare, hyperbilirubinemia, superior vena cava thrombus, and urethral stricture. The proportions of hemorrhagic and pulmonary complications were higher in the "intermediate" group than in the "low" group, although this difference did not reach statistical significance. The distribution of the remainder of complications including renal failure and urine leak were similar between the "low" and "intermediate" groups. Intraoperative complications included hemorrhage requiring transfusion, damage to an accessory renal artery, and injuries to the renal vein, vena cava and ureter (repaired laparoscopically on separate occasions), as well as injury to the common bile duct (repaired with an open operation).

A sub-analysis was carried out, including only those patients with "minor" complications. In this subgroup of patients, there was no difference between the median EBL or transfusion rate, although there was still a significant increase in WIT $(P<0.0001)$, LOS $(0.03)$ and operative times $(P<0.0001)$ with increasing nephrometry score. Thus, the association of higher nephrometry scores and longer WIT, LOS, and operative time cannot be solely attributed to a higher rate of major complications alone.

A sub-analysis evaluating individual surgical approaches (RAPN, CLPN and HAPN) was also carried out. Preoperative characteristics of BMI, preoperative eGFR and ASA were similar among tumor complexities in all subgroups, although patients in higher tumor complexity groups tended to be older in the RAPN and HAPN cohorts (data not shown). All groups showed significant increases in operative and ischemic time with increasing RENAL scores, with no significant differences noted in any of the individual groups for EBL, LOS, and the distribution of minor and major complications. Meanwhile, significant differences in complication rates were seen only in the RLPN subgroup; differences between this sub-analysis and the primary analysis might be attributed to limitations in sample size (Table 4).

The relationships between the individual scoring parameters and outcomes are shown in Table 5. Multivariate analysis revealed that the distance from the base of the tumor to the renal sinus was significantly associated with EBL, WIT, operative time and intraoperative complications. Larger tumor diameter significantly influenced EBL and operative time, whereas the anterior-posterior location significantly affected EBL and WIT. The location within the polar lines impacted WIT. The exophytic nature of the tumor was not independently linked to intraoperative outcomes. Among specific complications, distance to the renal sinus was associated with increased renal hemorrhage, with an odds ratio of 1.14 (1.00-1.30) per each one-point increase in nephrometry subscore for "distance to sinus", meaning closer distances to the renal sinus were associated with increased rates of renal hemorrhage. No single individual scoring parameter was predictive of any other specific complications.

Malignant tumors accounted for $69 \%, 96 \%$ and $88 \%$ of the "low", "intermediate" and "high" renal masses, respectively $(P=0.001)$. The distance to the renal sinus was associated with a higher rate of malignant tumors $(P=0.0006)$. Positive margin rates were not significantly different between groups (Table 2).

\section{Discussion}

The present study reviewed 298 patients undergoing MI-NSS for suspected malignancy, with the goal of further exploring the clinical role of the RENAL nephrometry system. Perioperative outcomes of EBL, WIT, OR time and LOS were found to increase with higher nephrometry scores. In addition, the proportion of major complications, as well as hemorrhage and respiratory complications, were associated with higher nephrometry scores. Finally, we have shown for the first time, to our knowledge, that individual components of the RENAL nephrometry score are predictive of outcomes, as summarized in Table 3.

The limitations of the present study deserve mention. Individual preferences in managing small renal masses might introduce bias. Tumor size and location dictate operative decision-making at our institution, ${ }^{13}$ with difficult tumors, especially those adjacent to the hilum, preferentially managed with RAPN. Additionally, institutional algorithms help guide the choice of treatment with active surveillance, extirpative therapy or ablation. Thus, patients presenting with criteria favoring active surveillance or ablation were 
Table 4 Sub-analysis of intra- and perioperative outcomes, stratified by RENAL nephrometry and surgical approach

\begin{tabular}{|c|c|c|c|c|c|}
\hline CLPN & & HAPN & & RAPN & \\
\hline Low $(n=84)$ & $\begin{array}{l}\text { Intermediate } \\
(n=67)\end{array}$ & Low $(n=9)$ & $\begin{array}{l}\text { Intermediate } \\
(n=26)\end{array}$ & Low $(n=42)$ & $\begin{array}{l}\text { Intermediate } \\
(n=62)\end{array}$ \\
\hline
\end{tabular}

\begin{tabular}{|c|c|c|c|c|c|c|}
\hline \multicolumn{7}{|l|}{ Intraoperative outcomes } \\
\hline EBL (mL) (median, [range]) & $150(20-1700)$ & $250(25-5000)$ & $300(100-500)$ & 450 (100-1400) & $150(5-1200)$ & $250(25-9000)$ \\
\hline WIT (min) & $10.8 \pm 13.9$ & $26.1 \pm 14.4$ & $10.7 \pm 9.4$ & $29.3 \pm 12.1$ & $22.4 \pm 9.3$ & $30.0 \pm 11.2$ \\
\hline Operative time (min) & $139 \pm 51$ & $184 \pm 64$ & $168 \pm 41$ & $210 \pm 46$ & $206 \pm 47$ & $230 \pm 55$ \\
\hline Transfusion requirement & $3(4 \%)$ & $4(6 \%)$ & $0(0 \%)$ & $6(23 \%)$ & $2(5 \%)$ & $4(6 \%)$ \\
\hline Positive margins & $6(11 \%)$ & $3(5 \%)$ & $1(14 \%)$ & $0(0 \%)$ & $4(13 \%)$ & $3(5 \%)$ \\
\hline \multicolumn{7}{|l|}{ Postoperative outcomes } \\
\hline LOS (days) & $2.2 \pm 1.5$ & $2.5 \pm 2.0$ & $3.0 \pm 1.6$ & $4.3 \pm 3.4$ & $2.5 \pm 1.8$ & $3.3 \pm 2.4$ \\
\hline eGFR & $84.7 \pm 24.4$ & $82.5 \pm 24.5$ & $81.7 \pm 28.7$ & $66.4 \pm 21.9$ & $85.2 \pm 22.1$ & $82.9 \pm 30.0$ \\
\hline eGFR $<60$ & $10(12 \%)$ & $11(16 \%)$ & $2(22 \%)$ & $7(27 \%)$ & $5(12 \%)$ & $9(15 \%)$ \\
\hline New onset eGFR $<60$ & $2(2 \%)$ & $5(8 \%)$ & $0(0 \%)$ & $4(15 \%)$ & $2(5 \%)$ & $3(5 \%)$ \\
\hline \multicolumn{7}{|l|}{ Complications } \\
\hline $\begin{array}{c}\text { \# Patients with } \geq 1 \\
\text { complication }\end{array}$ & $36(43 \%)$ & $28(42 \%)$ & $3(33 \%)$ & $15(58 \%)$ & $10(24 \%)$ & 31 (50\%) \\
\hline Minor complications & 32 (91\%) & $22(76 \%)$ & $4(100 \%)$ & $22(71 \%)$ & $13(93 \%)$ & $31(78 \%)$ \\
\hline Major complications & $3(9 \%)$ & 7 (24\%) & $0(0 \%)$ & 9 (29\%) & $1(7 \%)$ & 9 (22\%) \\
\hline
\end{tabular}

Highlighted outcomes indicate $P<0.05$ in comparisons between "Low" and "Intermediate" cohorts.

Table 5 Effect of individual components of RENAL nephrometry score on perioperative outcomes

$\begin{array}{llll} & \mathrm{R} & \mathrm{E} & \mathrm{EBL} \\ \mathrm{WIT}(\mathrm{mL}) & \\ \text { Operative time (min) }\end{array}$

Arrows indicate statistical significance on multivariable analysis. Up arrows indicate an increase in outcome with higher RENAL nephrometry scores. The down arrow indicates a decrease in outcome as associated with anterior location. Black arrows: $P<0.0001$. Gray arrows: $P$ range 0.0001-0.01. White arrows: $P$ range 0.01-0.05. A, Anterior/ Posterior location; E, Exophytic nature; L, Location within Polar Lines; N, Nearness to Renal Sinus; R, Radius.

not included in the present analysis. Furthermore, at our institution, the most difficult tumors tend to be managed with open NSS, as evidenced by the low proportion of "high" complexity tumors in our study of MI-NSS. The distribution of nephrometry scores in our series, however, is similar to that reported in the literature of MI-NSS. ${ }^{7}$ Our high rate of malignancy is likely explained by our heavy bias towards renal biopsy. However, these results are more applicable to the intended treatment of small renal masses, and represent the expected outcomes of those patients that require treatment.

Unmeasured variables might also introduce bias into our results. Variations in surgeon technique, for instance, will affect perioperative outcomes. The surgeon effect in our series is difficult to determine given individual preferences regarding surgical approach. Included in this review were conventional laparoscopic and robotic approaches. Despite the heterogeneous nature of this group, studies from our group and others have shown few differences in outcomes between these approaches. ${ }^{13,14}$ Furthermore, on sub-analysis among these separate approaches, there did not appear to be a single surgical approach whose outcomes "drove" the overall differences seen. Thus, we feel that our findings are indicative of a diverse academic practice and perhaps, as a result, are a more rigorous assessment of the utility of the nephrometry system.

These limitations notwithstanding, we believe our series provides an important assessment of the RENAL nephrometry scoring system in a heterogeneous, multi-surgeon practice, in what is to our knowledge, the largest external validation of the RENAL scoring system for MI-NSS. The present findings corroborate previous reports correlating the RENAL system to perioperative outcomes, complications and pathological features of small renal masses. ${ }^{7,15}$ No previous studies evaluated the importance of individual param- 
eters of the RENAL system, as reported here. The RENAL nephrometry score has been shown to be predictive of surgical approach, with higher complexity tumors more likely to receive open nephron-sparing or minimally invasive nonnephron sparing surgery. ${ }^{16}$ However, recent data suggest high complexity tumors can have similar outcomes with both open and robotic partial nephrectomy. ${ }^{17}$ In this context, the present findings regarding the importance of individual RENAL parameters might serve to aid in risk-stratifying patients with small renal masses, augmenting the total score as well as existing gauges of comorbidity, such as performance status and the Charleston Comorbidity Index. These findings might encourage more surgeons to pursue renal mass biopsy, active surveillance or referral to high volume surgeons for extirpative therapy for more complex renal masses. Furthermore, surgeons might use these findings to better inform patients about the risks inherent to their particular tumor. Certain factors, such as tumor diameter and depth of invasion, appear to be more predictive of outcomes, and these components might merit differential weighting to further define patient-specific risk. Ultimately, however, it is important to note that the present results confirm that higher complexity tumors are amenable to nephron-sparing surgery, albeit with greater yet acceptable perioperative risks.

The future role of the RENAL scoring system in clinical and investigative urology is yet to be solidified. The longterm impact of nephron-sparing surgery for complex masses with regards to oncological control and renal function still warrants additional investigation. Better understanding the effects of individual renal mass anatomical characteristics might allow for further refinement of the scoring system. Furthermore, multiple nephrometry scores, including the RENAL score, Preoperative Aspects and Dimensions Used for Anatomic classification and C-index have been proposed for grading of tumor complexity. As limited data exist comparing these instruments, it remains unknown which scoring system will gain traction in a clinical setting. However, the RENAL system does have the advantage of being simple to implement and reproducible. ${ }^{6}$ Based on the results presented here, we believe the RENAL nephrometry system is useful for surgeons for both preoperative and intraoperative decision-making.

Thus, more complex tumors are associated with longer operative times, warm ischemia times and greater blood loss, as well as a higher proportion of severe complications. The distance from the tumor to the renal sinus appears to be the most influential individual component of the RENAL system, although tumor diameter, polarity and anteriorposterior location also have significant effects on outcomes. The ultimate clinical and research role of current nephrometry scoring systems remains to be seen, but the RENAL system is useful for patient counseling, operative planning and as a research instrument.

\section{Acknowledgment}

We acknowledge Rick Saur for maintenance of the partial nephrectomy database.

\section{Conflict of interest}

None declared.

\section{References}

1 Gill IS, Kavoussi LR, Lane BR et al. Comparison of 1,800 laparoscopic and open partial nephrectomies for single renal tumors. J. Urol. 2007; 178: 41-6.

2 Wheat JC, Roberts WW, Hollenbeck BK, Stuart Wolf Jr J, Weizer AZ. Complications of laparoscopic partial nephrectomy. Urol. Oncol. 2011; doi: 10.1016/j.urolonc.2010.11.003.

3 Kutikov A, Uzzo RG. The R.E.N.A.L. nephrometry score: a comprehensive standardized system for quantitating renal tumor size, location and depth. J. Urol. 2009; 182: 844-53.

4 Hew MN, Baseskioglu B, Barwari K et al. Critical appraisal of the PADUA classification and assessment of the R.E.N.A.L. nephrometry score in patients undergoing partial nephrectomy. J. Urol. 2011; 186: 42-6.

5 Simhan J, Smaldone MC, Tsai KJ et al. Objective measures of renal mass anatomic complexity predict rates of major complications following partial nephrectomy. Eur. Urol. 2011; 60: 724-30.

6 Rosevear HM, Gellhaus PT, Lightfoot AJ, Kresowik TP, Joudi FN, Tracy CR. Utility of the RENAL nephrometry scoring system in the real world: predicting surgeon operative preference and complication risk. BJU Int. 2012; 109: 700-5.

7 Hayn MH, Schwaab T, Underwood W, Kim HL. RENAL nephrometry score predicts surgical outcomes of laparoscopic partial nephrectomy. BJU Int. 2011; 108: 876-81.

8 Mufarrij PW, Krane LS, Rajamahanty S, Hemal AK. Does nephrometry scoring of renal tumors predict outcomes in patients selected for robot-assisted partial nephrectomy? J. Endourol. 2011; 25: 1649-53.

9 Kolla SB, Spiess PE, Sexton WJ. Interobserver reliability of the RENAL nephrometry scoring system. Urology 2011; 78: $592-4$.

10 Weight CJ, Atwell TD, Fazzio RT et al. A multidisciplinary evaluation of inter-reviewer agreement of the nephrometry score and the prediction of long-term outcomes. J. Urol. 2011; 186: 1223-8.

11 Dindo D, Demartines N, Clavien PA. Classification of surgical complications: a new proposal with evaluation in a cohort of 6336 patients and results of a survey. Ann. Surg. 2004; 240: 205-13.

12 Levey AS, Greene T, Schluchter MD et al. Glomerular filtration rate measurements in clinical trials. Modification of Diet in Renal Disease Study Group and the Diabetes Control and Complications Trial Research Group. J. Am. Soc. Nephrol. 1993; 4: 1159-71. 
13 Weizer AZ, Gilbert SM, Roberts WW, Hollenbeck BK, Wolf JS Jr. Tailoring technique of laparoscopic partial nephrectomy to tumor characteristics. J. Urol. 2008; 180: 1273-8.

14 Haber GP, White WM, Crouzet S et al. Robotic versus laparoscopic partial nephrectomy: single-surgeon matched cohort study of 150 patients. Urology 2010; 76: 754-8.

15 Kutikov A, Smaldone MC, Egleston BL et al. Anatomic features of enhancing renal masses predict malignant and high-grade pathology: a preoperative nomogram using the RENAL Nephrometry score. Eur. Urol. 2011; 60: 241-8.

16 Canter D, Kutikov A, Manley B et al. Utility of the R.E.N.A.L nephrometry scoring system in objectifying treatment decision-making of the enhancing renal mass. Urology 2011; 78: 1089-94.

17 Simhan J, Smaldone MC, Tsai KJ et al. Perioperative outcomes for robotic and open partial nephrectomy in moderately and highly complex renal lesions. J. Urol. 2012; 187: $2000-4$.

\section{Editorial Comment}

\section{Editorial Comment from Dr Herrel and Dr Canter to Association of RENAL nephrometry score with outcomes of minimally invasive partial nephrectomy}

The RENAL nephrometry scoring system as first introduced by Kutikov and Uzzo offers an objective measurement of the salient factors contributing to the anatomical complexity of a given renal mass. ${ }^{1}$ This tool creates a common language to allow comparison of surgical techniques, complications, pathological diagnosis and cancer control outcomes. In addition to its predictive power, the RENAL nephrometry scoring system is highly reproducible and easy to use. Since its introduction, there have been over 80 peer-reviewed publications in which the RENAL nephrometry system has been used to standardize outcome reporting stratified by tumor complexity.

For example, Simhan et al. showed that more complex tumors as quantified by a higher nephrometry score have increasing rates of major urological complications in patients undergoing nephron-sparing surgery. ${ }^{2}$ More recently, Canter et al. showed that the nephrometry score of a patient's tumor predicted the choice of surgical procedure; patients with more complex tumors as objectified by a higher nephrometry score were more likely to undergo radical nephrectomy. ${ }^{3}$ Similarly, patients with a low nephrometry score were more likely to undergo minimally invasive nephron-sparing surgery.

In the article contained in this issue of the International Journal of Urology, the authors implement the RENAL nephrometry scoring system to stratify outcomes in patients undergoing minimally invasive (pure laparoscopic or robotic) partial nephrectomy. ${ }^{4}$ The authors showed the predictive ability of not only the nephrometry score sum, but also the individual components of the scoring system. Patients with increasingly complex tumors as quantified by a higher nephrometry sum, as well as increasing individual component scores, tended to have longer warm ischemia times and length of stays, increased estimated blood loss intraoperatively, and an increase in major complication rates. In particular, in this study, the " $\mathrm{N}$ " variable, or nearness to the collecting system, had the greatest association with outcomes.

The utility of objective tools to predict outcomes cannot be overstated. Statistical models and objective measures offer physicians the ability to more precisely counsel patients on the risks, complications and outcomes associated with intervention on their disease process, thereby helping to guide treatment decision-making. The goal of tools such as the RENAL nephrometry scoring system is to replace subjective "hunches" with objective decision-making to improve patient care.

Lindsey A Herrel M.D. and Daniel Canter M.D. Department of Urology, Emory University, Atlanta, Georgia, USA daniel.j.canter@emory.edu

DOI: 10.1111/iju.12006

\section{Conflict of interest}

None declared.

\section{References}

1 Kutikov A, Uzzo RG. The R.E.N.A.L. nephrometry score: a comprehensive standardized system for quantitating renal tumor size, location and depth. J. Urol. 2009; 182: 844-53.

2 Simhan J, Smaldone MC, Tsai KJ et al. Objective measures of renal mass anatomic complexity predict rates of major complications following partial nephrectomy. Eur. Urol. 2011; 60: 724-30.

3 Canter D, Kutikov A, Manley B et al. Utility of the R.E.N.A.L. nephrometry scoring system in objectifying treatment decision-making of the enhancing renal mass. Urology 2011; 78: 1089-94.

4 Ellison JS, Montgomery JS, Hafez KS et al. Association of RENAL nephrometry score with outcomes of minimally invasive partial nephrectomy. Int. J. Urol. 2013; 20: 564-70. 